\title{
La luce «turneriana» dei paesaggi padani
}

Filippo Milani

\section{(2) OpenEdition}

\section{Journals}

Edizione digitale

URL: https://journals.openedition.org/cher/683

DOI: 10.4000/cher.683

ISSN: 2803-5992

\section{Editore}

Presses universitaires de Strasbourg

\section{Edizione cartacea}

Data di pubblicazione: 9 juillet 2020

Paginazione: 57-67

ISBN: 979-10-344-0068-3

ISSN: 1968-035X

\section{Notizia bibliografica digitale}

Filippo Milani, «La luce «turneriana» dei paesaggi padani», reCHERches [Online], 24 | 2020, online dal 20 septembre 2021, consultato il 19 novembre 2021. URL: http://journals.openedition.org/cher/683 ; DOI: https://doi.org/10.4000/cher.683 


\title{
La luce «turneriana» dei paesaggi padani
}

\author{
FILIPPO MILANI
}

$\mathrm{N}^{\prime}$

ell'intervento dal titolo "Di cosa si parla quando si parla di paesaggio", presentato al convegno Il paesaggio come capitale (tenutosi a Reggio Emilia nel 2006), Gianni Celati per riflettere sulla dissoluzione odierna del rapporto uomo-natura fa riferimento alla capacità - ormai perduta - di saper guardare il mondo circostante con naturalezza e compartecipazione che è la caratteristica precipua della pittura paesaggistica, dai vedutisti del Settecento a Cézanne. In particolare, egli afferma che:

artisti come Caspar Friedrich, Turner, Constable, Cézanne, hanno lavorato per risensibilizzare la percezione, per riaprirla alla momentaneità incatturabile d'ogni sguardo, e ritrovare la primitività d'ogni paesaggio, come spazio d'iniziazione all'esperienza estatica. (Celati, 2008: s. p.)

Non si tratta di una riscoperta del «pittoresco» ma di un modo differente di entrare in relazione con il paesaggio, che invita a riattivare la «primitività» del rapporto uomo-natura, risensibilizzando lo sguardo alla percezione fenomenologica del mondo. Il grande equivoco contro cui si oppone Celati è l'effetto di estrema idealizzazione subito dal paesaggio negli ultimi due secoli, poiché esso ormai - se ne è reso conto per primo Cézanne - viene osservato come se fosse già un quadro, una porzione di realtà preconfezionata, ricorrente soprattutto nella figurazione del paesaggismo letterario di quello che egli chiama il «qui si vede questo». La sovrapposizione tra paesaggio reale e idealizzato conduce a una tale assuefazione estetica che il paesaggio viene depotenziato in quanto «spazio d'iniziazione dell'esperienza estatica» e si riduce a mero sfondo decorativo. L'adeguamento al «già noto» impone allo sguardo un «guardare categoriale», «dove al posto delle percezioni ci sono categorie pronte a congelare ogni movimento dello sguardo, e sostituire ogni incertezza delle percezioni, con una supposta oggettività della cosa guardata» (Celati 2008: s. p.), ovvero categorie scopiche che impediscono di fare esperienza diretta della visione, anche nella sua dimensione effimera di illusione del reale, perché esse filtrano lo sguardo

1 Filippo Milani, Università di Bologna. 
attraverso valori culturali già predefiniti senza lasciare spazio al rapporto intimo con l'esterno. Allora, per riuscire a guardarlo con altri occhi è necessario - come consiglia Celati ai nuovi paesaggisti - «togliere di mezzo ciò che si è già costituito in concetto, in definizione categoriale, che obnubila lo sguardo, e che in realtà non ti lascia vedere niente perché "sai già tutto"»(Celati 2008: s. p.).

Tale riflessione rivela notevoli affinità con gli studi del critico d'arte bolognese Francesco Arcangeli (Bologna, 1915-1974), allievo diretto di Roberto Longhi, che negli anni Cinquanta e Sessanta propose il fondamentale concetto di "naturalismo informale», individuando in Turner il progenitore non solo dell'impressionismo (Monet) e del post-impressionismo (Cézanne) ma anche dell'arte informale (da Fautrier a Pollock, da Wols a Morlotti), per essere riuscito a mostrare attraverso il particolare trattamento dei colori la vitalità della componente non-formale della forma che incide sulla rappresentazione del paesaggio e sulla percezione soggettiva del reale ${ }^{2}$. Il riferimento ad Arcangeli non è casuale ma si fonda sulla formazione bolognese dello scrittore che - come ricostruisce la biografia inserita nel Meridiano Mondadori - compì i suoi studi proprio nel capoluogo felsineo. Si iscrisse prima al Liceo classico «L. Galvani», che abbandonò in breve tempo, per poi trasferirsi al Liceo classico «M. Minghetti», dove portò a termine gli studi, diplomandosi nel 1958, e dove in quegli stessi anni insegnava proprio Arcangeli - in una sezione diversa ${ }^{3}$. Anche se non è possibile ricostruire se ci siano stati rapporti diretti tra i due, di certo sappiamo che Celati strinse particolari rapporti di amicizia con alcuni dei suoi professori del «Minghetti» (ad esempio, il professore Festi che insegnava filosofia) ed era interessato alle ricerche storico-artistiche di Longhi, perciò potrebbe aver seguito qualche conferenza di Arcangeli sia al liceo sia all'Accademia di Belle Arti dove egli esercitava la libera docenza negli anni Cinquanta e poi all'Università negli anni Sessanta, quando lo scrittore si iscrisse alla Facoltà di Lettere.

Dunque, non è così azzardato riscontrare una certa affinità tra le rispettive riflessioni parallele sulle trasformazioni che contraddistinguono la rappresentazione del paesaggio nella contemporaneità. Di conseguenza, si intende procedere all'analisi - avvalendosi anche degli strumenti della critica storico-artistica, in particolare di quella arcangeliana - della descrizione dei paesaggi padani in Narratori delle pianure (1985), Quattro novelle sulle apparenze (1987), Verso la foce (1989), con l'obiettivo di delineare il nuovo

2 Un percorso intellettuale che alcuni anni fa è stato riscoperto anche grazie alla mostra Turner Monet Pollock: dal romanticismo all'informale. Omaggio a Francesco Arcangeli, catalogo della mostra tenutasi al Museo d'arte della città di Ravenna, 19 marzo-23 luglio 2006, a cura di C. Spadoni, Milano, Electa, 2006.

3 La consultazione degli annuari dei due licei ha consentito di verificare che Celati si iscrive nell'anno 1952-53 alla IV ginnasio sezione $\mathrm{D}$ del «Galvani» ma viene bocciato a fine anno con insufficienze in quasi tutte le materie; mentre l'anno scolastico successivo si iscrive alla sezione D del «Minghetti», dove si diploma nel 1958. Per la consultazione degli annuari si ringraziano rispettivamente Verena Gasperotti («Galvani») e Agnese Serra («Minghetti»). 
rapporto sentimentale e visivo che Celati tenta di instaurare con un paesaggio che, seppur deturpato dallo sfruttamento industriale degli spazi, può ancora offrire una certa «momentaneità incatturabile», impressioni o almeno macchie informi di luce «naturale», come nella pittura turneriana.

I paesaggi narrati da Celati negli anni Ottanta, attraverso la sollecitazione degli sguardi di amici fotografi, risultano intrisi di luce, sia «naturale» che artificiale, ma essa ritaglia forme che oscillano tra nitidezza dell'immagine bloccata nel tempo e disintegrazione del paesaggio che lo sguardo non riesce ad afferrare completamente. Camminando immerso nell'atmosfera densa della pianura padana, tra nebbia, polvere e smog, Celati percepisce quanta continuità ci sia tra la razionalizzazione della divisione in lotti del territorio e la «disfazione» luminosa: il paesaggio è tanto più suddiviso in maniera geometrica da strade perpendicolari, capannoni cubici e villette geometrili, quanto più diviene impossibile da vedere, letteralmente «in-guardabile», perché l'osservatore è sempre immerso in un vortice luminoso destabilizzante. Per questo, nelle descrizioni paesaggistiche celatiane viene messo in evidenza il rapporto compromesso e inaridito tra uomo e natura, cosicché anche nei rari momenti in cui il paesaggio sembra "visibile», esso scivola immediatamente nell'indistinto, nell'indecifrabile (come nell'acquerello di Turner Colour Beginning, 1819, nel quale si cerca di cogliere il momento esatto in cui la luce al mattino fa nascere il colore dal buio ma senza ancora poter distinguere cielo, terra e gli altri elementi del paesaggio).

Il narratore è combattuto tra la volontà di fissare (come in uno scatto fotografico) momenti di vita che sembrano riannodare i fili con il paesaggio, vissuto in maniera simbiotica ma non idealizzato, e la consapevolezza di quanto il dato esterno risulti inafferrabile, sia con lo sguardo sia tramite la scrittura. La necessità di ritrovare una partecipazione sensibile nel rapporto con i paesaggi padani era già stata espressa da Ezio Raimondi, nella premessa al volume che raccoglie gli atti del convegno L'orizzonte di bruma. Luoghi del Novecento poetico in Emilia (2002), proprio a partire da una formula arcangeliana:

nel momento in cui anche la provincia diviene una provincia del mondo, per riprendere la formula appassionata e lungimirante di Francesco Arcangeli, ancora alle soglie degli anni '60, il «luogo» acquista di nuovo valore, è il «qui» e «ora» di un legame vitale che non separa ma unisce, quanto più invita alla concretezza di una visione diretta in cui leggere equivale anche a guardare, sentirsi immerso nel mondo, vicino e insieme lontano. [...] acquista una evidenza singolare come un paesaggio illuminato di colpo in tutti i suoi angoli d'ombra, ed ecco che la scena si anima di eventi, figure, incontri, episodi, amicizie, progetti, libri, posti, ambienti, riviste, con una sorta di colore comune, di atmosfera corale tra voce e aria. (Raimondi 2002: 5)

La necessità di ritrovare un legame nuovamente vitale con il «luogo», grazie al quale guardare in maniera diversa le cose, sentendosi immersi nel mondo e nelle sue contraddizioni, consente di recuperare storie e immagini che altrimenti andrebbero perdute. Tale riflessione è del tutto in sintonia con l'operazione 
narrativa compiuta da Celati, come testimonia il racconto intitolato Ultimi contemplatori (pubblicato proprio in appendice al medesimo volume) e in cui si legge:

Nelle grandi pianure del Po, l'occhio non si perde mai nello spazio aperto, non è mai colto di sorpresa da conformazioni impreviste del paesaggio, e piuttosto a poco a poco comincia a riconoscere un grande ordine astratto come quello dell'architettura. (Celati 2002a: 203)

Lo spazio della pianura padana si configura paradossalmente non come uno spazio aperto, illimitato, ma come una spazio dominato da un principio ordinatore naturale, quasi architettonico, anche se latente. Celati continua distinguendo il valore della contemplazione da quello del «pittoresco naturale» in un paesaggio apparentemente arido e privo di interesse, ma in realtà profondamente mutevole perché legato agli umori di un fiume tanto invisibile quanto imprevedibile:

Nelle nostre pianure l'immagine della natura è sempre stata legata alle tremende piene del Po, all'esuberanza distruttiva delle acque, all'enorme quantità di falde freatiche dove le acque penetrano facilmente attraverso i terreni argillosi, sbucando all'impensata in polle, stagni, paludi, o risorgive. [...] Per questo non esiste dalle nostre parti un'idea della natura riposante e idillica come in altri paesi. Qui le acque mostrano la natura come qualcosa che non è assoggettabile al controllo dell'uomo, come una esuberanza imprevedibile, come una pazzia a cui gli uomini debbono adattarsi. (Celati 2002a: 207)

Il concetto di natura come «esuberanza imprevedibile» richiama in qualche modo lo sguardo romantico, non inteso come afflato sentimentale dell'io verso la natura ma come soggetto destabilizzato dall'incomprensibile accadere della natura, "come una pazzia a cui gli uomini debbono adattarsi». Per Celati gli «ultimi contemplatori» sono coloro che non si limitano alla descrizione del paesaggio visibile ma sono alla ricerca di nuovi modi per entrare in relazione con gli spazi, mossi da quella che egli chiama - nel saggio "Collezione di spazi» del 2003 - «l'urgenza di aprire nuove visioni dello spazio», e che egli individua nei maggiori rappresentanti dell'arte e della letteratura cosiddette «moderne»:

Dal nuovo paesaggismo di Constable e Turner, da Poe a Melville, da Baudelaire a Rimbaud, fino a Nietzsche e Rilke, c’è una ricerca sul modo di pensare lo spazio esterno, e l’urgenza di altre visioni dello spazio. Lo spazio è la figura di qualcosa che si apre là davanti a noi, anche al di là della linea d'orizzonte, anche al di là del visibile. (Celati 2003: 58)

Alcuni artisti e scrittori della modernità hanno sentito l'urgenza di rappresentare lo spazio esterno anche al di là delle categorie ermeneutiche razionalistiche che si limitano a constatare l'impossibilità logica di vedere oltre l'orizzonte, ma hanno esteso lo sguardo oltre il visibile, grazie all'immersione totale nell'ambiguità percettiva del sensibile. Per questa ragione si può rintracciare una corrispondenza con il noto saggio di Arcangeli del 1972, «Lo spazio romantico», scritto in occasione della mostra parigina sul Romanticismo inglese, nel quale lo storico dell'arte bolognese afferma che l'arte romantica - 
in particolar modo la pittura di Turner (con particolare riferimento al quadro Norham Castle: sunrise, 1835-1840) - è caratterizzata da "qualche cosa di inafferrabile e di ambiguamente definibile entro uno spazio non più definito prospetticamente», e di conseguenza lo spazio romantico non si configura come la ricognizione del visibile ad una distanza determinata tramite il filtro di una coscienza intellettuale ma «è uno spazio che può arrischiare l'indagine, e l'intuizione in immagini, dell'immensità dell'universo non più autocentrata dall'uomo proprio perché, nello stesso tempo, presuppone una esplorazione interiore che non conosce limiti» (Arcangeli 1977: 9-11).

Il realismo di Turner, per quanto paradossale, risiede nella volontà di mostrare il dato reale attraverso il filtro immediato della percezione, senza la sovrastruttura aprioristica della decodificazione razionale che impone la cristallizzazione delle forme e della luce, restringendo l'ampia gamma del campo visivo: così nella pittura dell'ultimo Turner «la forma sembra casuale, e lo spazio è invaso da larve, da labili macchie senza collocazione prefissa, Turner, insomma, spalancando totalmente lo spazio fin quasi a distruggerlo, sfocia, come concezione e significato, nell'informale» (Arcangeli 1976: 10-14); come ad esempio in Light and Colour (Goethe's Theory) - The Morning after the Deluge, 1843. Infatti, nonostante in Turner sembri mancare qualsiasi aderenza alla realtà - così come nell'arte informale - conducendo ad una apparente disintegrazione nella relazione tra esperienza e immaginazione, in realtà il pittore inglese fa sempre affidamento sulla percezione sensoriale dello spazio circostanze e della variazione delle condizioni atmosferiche; come ha puntualizzato Sir Kenneth Clark nel suo fondamentale saggio Il paesaggio nell'arte ${ }^{4}$, ricordando un aneddoto legato alla composizione del dipinto Rain, Steam and Speed (1844):

il [quadro di] Turner pare una fantasia poetica, senza alcuna relazione con l'esperienza. Ma ci smentisce la testimonianza di Mrs Simon. Costei si trovava in treno, durante una pioggia torrenziale, quando, con sua grande sorpresa, vide un gentile signore anziano, seduto di fronte a lei, sporgere la testa fuori dal finestrino e restare così per circa nove minuti. Costui poi si ritrasse, grondante d'acqua, e tenne chiusi gli occhi per un quarto d'ora. Nel frattempo la giovane signora, incuriosita, mise a sua volta la testa fuori dal finestrino e, naturalmente, si inzuppò d'acqua, ma fece un'indimenticabile esperienza. Immaginiamo la sua gioia quando, all'esposizione dell'Accademia dell'anno seguente, si trovò di fronte Pioggia, vapore e velocità, e udendo qualcuno che, con tono infastidito diceva: «E proprio da Turner, non è vero? Chi mai ha visto una farragine tanto assurda?», poté ribattere «Io l'ho vista». (Clark 1985: 153-154)

Questo aneddoto consente di riconsiderare la rappresentazione turneriana del rapporto tra esperienza e immaginazione, perché rivela come l'interesse per l'immaterialità luminosa sia imprescindibile dall'aderenza alla percezione del dato reale. Anche Celati, rifacendosi alle parole di Ruskin su Turner, osserva come il pittore inglese sia stato determinante per considerare lo spazio come un

4 Prima edizione del 1949, tradotto in italiano nel 1962. 
campo fenomenico al di fuori di noi, dove agisce una luce inafferrabile: «le sue pennellate seguono lampi di colore in cui sfuma ogni forma definita; e le cose spuntano vaghe da intensità abbaglianti di squarci luminosi» (Celati 2003: 70).

L'intuizione di Arcangeli è tendere un filo rosso tra il pittore inglese e la pittura informale, individuando la medesima volontà di ribaltare il regime scopico dominante di epoca in epoca, che impedisce di vedere il mondo nella sua complessità. Si tratta di una sotterranea ma costante rivoluzione copernicana, che dagli artisti romantici passa agli impressionisti fino a giungere agli informali; come ha rilevato ancora Kenneth Clark, collocando la svolta impressionista all'interno della storia dell'arte occidentale:

L'impressionismo riscoprì la luce. [...] ci ha dato qualcosa che è sempre stata una delle grandi conquiste dell'arte: ha allargato il nostro orizzonte visivo. [Gli impressionisti] ci insegnarono a vedere il colore nelle ombre. Ogni giorno ci fermiamo con gioia a contemplare un effetto di luce che altrimenti sarebbe rimasto inosservato. (Clark 1985: 144-145)

Quest'ultima frase risulta particolarmente calzante per individuare la spinta narrativa che sta alla base della scrittura di Celati dagli anni Ottanta in poi, ovvero a partire dalla collaborazione con Luigi Ghirri, perché la descrizione del paesaggio padano è volta proprio alla gioiosa contemplazione di particolari apparentemente irrilevanti che offrono prospettive inattese sul quotidiano, appunto riuscendo a cogliere «un effetto di luce che altrimenti sarebbe rimasto inosservato».

In questa prospettiva, si possono analizzare le rappresentazioni del paesaggio in Narratori delle pianure, Quattro novelle sulle apparenze e Verso la foce; rappresentazioni che si delineano come microsistemi in cui lo sguardo è immerso nel paesaggio - inscape secondo l'espressione di Gerard M. Hopkins - quello deturpato della provincia padana ma allo stesso tempo offre una percezione organica della luce, anche se marginale, frammentaria e dispersiva. Lo sguardo celatiano sul paesaggio è un tentativo di togliere la sovrastruttura del pittoresco alla natura - come accade in Turner - e la patina dell'abitudinario agli oggetti del quotidiano - come accade in Morandi. Celati lo esplicita con puntualità nell'intervento "Un sistema di racconti sul mondo esterno», presentato al seminario La rappresentazione dell'esterno (Reggio Emilia, 23-24 novembre 1984) e poi pubblicato sul numero monografico della rivista Quindi nel gennaio 1986: «Dare per scontato qualcosa significa rinunciare a descriverlo. Noi diamo per scontato qualcosa in base a delle presupposizioni, e le presupposizioni sono appunto l'opposto delle descrizioni» (Celati 1986: 6). Perciò, la scrittura stessa, ovvero la rappresentazione del mondo esterno attraverso le parole, può avere ancora senso, solo se riesce ad intercettare eventi accidentali e incomprensibili (un incontro, una chiacchiera, una veduta inattesa), nonostante l'ordine imposto allo sguardo dalla lottizzazione sfrenata dello spazio e dall'inaridimento dell'immaginario: «ciò che ha senso - continua Celati - è il fatto che là fuori vi sia un ordine, con confini da tutte le parti, oltre i quali c'è un orizzonte di eventi incomprensibili» (Celati 1986: 9). 
Così, ad esempio, nei brevi racconti di Narratori delle pianure, risulta evidente l'attenzione posta da Celati alla necessità di riattivare uno sguardo non scontato, un continuo ribaltamento copernicano in contrapposizione al miope regime scopico imposto dall'architettura industriale, dalla moltiplicazioni delle infrastrutture e dalla cartellonistica pubblicitaria (un ribaltamento messo in evidenza anche dalla efficace copertina di Esplorazioni sulla Via Emilia, 1986). Il ribaltamento è legato innanzitutto all'impedimento della visione come nel racconto Bambini pendolari che si sono perduti, quando i bambini protagonisti si disorientano immersi nella densità bianca della nebbia padana:

Appena fuori da un altro quartiere non vedevano più niente, dovevano essere in aperta campagna, stavano attraversando campi gelati, e intorno era tutto bianco: una nebbia così bianca come non l'avevano mai vista, ma anche così fitta che dovevano frugare col piede il terreno davanti a loro prima di fare un passo, perché non vedevano niente oltre il loro naso. (Celati 2016: 751)

La nebbia è una luce bianca che non illumina la via ma, al contrario, impedisce allo sguardo di trovare punti di riferimento, ed è proprio a partire dall'osservazione compromessa e imperfetta che si può ripartire per guardare il paesaggio, per «ri-sentire» i luoghi. Per compiere questa operazione di pulizia dello sguardo, Celati sente la necessità di recuperare anche alcune memorie familiari alla ricerca di un paesaggio padano ancora integro; come accade nel racconto Traversata delle pianure, compiuta dalla madre agli inizi del Novecento per trasferirsi dalla campagna alla città, nel quale emergono colori ormai scomparsi attraverso lo sguardo disorientato dei parenti del narratore che cercano di capire da che parte tramonta il sole - scoprendo che Porta Mare si trova ad est e non a ovest:

hanno tenuto d'occhio la prospettiva di Porta Mare, per vedere se il sole riusciva ad andar giù da quella parte. I colori della strada che avevano di fronte dovevano essere tutti smorzati, nessuno dei colori netti che conosciamo noi; dovevano esserci sfumature di ocra e seppia e terra di Siena nell'intonaco delle case, il colore del mattone vecchio in una chiesa, il grigio polveroso dell'acciottolato fino in fondo alla strada. (Celati 2016: 814)

I colori ricordano quelli di un dipinto paesaggista di fine Ottocento (si pensi a Fontanesi ma anche, a livello locale, a Luigi Bertelli o Alessandro Scorzoni) in una amalgama tenue e uniforme di toni «tutti smorzati», senza interferenze di colori decisi che staccano con il contesto, come invece accade oggi. Inoltre, la gamma dei colori e l'atmosfera polverosa richiama evidentemente i dipinti di Giorgio Morandi, sia i paesaggi sia le nature morte, verso i quali è nota l'ammirazione di Celati, in prima istanza grazie agli insegnamenti di Roberto Longhi e in seguito attraverso lo sguardo fotografico di Luigi Ghirri (che ha anche ritratto lo studio del pittore in via Fondazza a Bologna).

Si tratta di colori irrecuperabili, perché ormai in un paesaggio che subisce la pressione del neocapitalismo l'osservazione è impedita non solo dagli agenti atmosferici ma soprattutto dalla moltiplicazione dei tralicci di metallo a fini promozionali, creando un contrasto «tra la dimensione orizzontale, poetica, 
delle nuvole, e la verticalità sfrontata dei pannelli pubblicitari, che parlano una lingua involontariamente comica» (Iacoli 2002: 71) ${ }^{5}$. Basti pensare al Paesaggio con centrale nucleare in Verso la foce: "Cirri biancastri a grande altezza, filamenti di nubi ferme per assenza di vento. Sullo stradone camion e camion che mi sventagliano con folate violente, e moltissimi cartelli pubblicitari: BRIO RUBINETTI, ABC CUCINA, IL PORCELLINO CARNE FRESCA DI MAIALE, CASITALIA PREFABBRICATI, ALFIERI DANIELE CAMINETTI E RIVESTIMENTI» oppure, sempre nello stesso racconto, «Sforzo per proseguire, ogni momento in avanti è spazio vuoto, tempo vuoto da colmare. Almeno non ci fossero tutti quei cartelli pubblicitari, un numero sconfinato di parole che mi dà la depressione» (Celati 2016: 1002 e 1011); ma anche al racconto Come fa il mondo ad andare avanti, contenuto in Narratori delle pianure, in cui il protagonista si accorge "di non poter più mettere gli occhi quasi da nessuna parte senza trovare delle parole stampate da leggere» (Celati 2016: 774). Quando lo sguardo riesce ad oltrepassare la selva di cartelli pubblicitari, allora intravede un paesaggio "naturale» che non riesce più a comprendere, un accumulo di elementi eterogenei che sfociano in un magma informale; come accade ne Il ritorno del viaggiatore, dove la descrizione di una waste land padana sembra intersecare l'indefinito turneriano di The Evening Star (1830), l'angoscia del Cane interrato (1821) di Goya e la matericità dell'arte informale di Fautrier:

Dall'altra parte questo paese si apriva verso terreni devastati; macerie, fino ad un punto lontano dove vedevo solo sassi e mota. Sembrava d'essere in un avamposto, dei cani rovistavano in un mucchio di spazzatura. (Celati 2016: 831)

L'osservatore non scava oltre la superficie dell'apparenza, ma prende coscienza delle contraddizioni del reale di cui egli fa esperienza nello sguardo ancor prima che attraverso una puntuale riflessione. Si tratta di «topografie dentellate», di paesaggi caratterizzati da un affollamento di elementi eterogenei che destabilizzano ma - puntualizza Iacoli - «possiedono tutti una forma di sospensione, di incanto che li trattiene, come nella fissità di una cartolina, in bilico tra uno stato di grazia e una silenziosa rassegnazione» (Iacoli 2002: 73). Sia la riconfigurazione del paesaggio legata allo sviluppo industriale e alla sua decadenza sia la persistenza di elementi «naturali» vengono osservati sullo stesso piano, come aspetti inscindibili che soggiacciono all'ineluttabile legge della trasformazione. É come se tutti i personaggi dei racconti si sentissero per la prima volta immersi nel paesaggio senza sovrastrutture difensive, prospettive precostituite, punti d'osservazione preconfezionati che impongono una colorazione ben definita e artificiale al paesaggio; invece per un momento si sentono tutti «allo scoperto» come l'ex detenuto guercio protagonista dell'omonimo racconto, Allo scoperto:

5 A questo proposito è interessante fare riferimento al progetto fotografico Visible Distance/Second Sight dell'artista statunitense Jennifer Bolande che nel 2017 ha sostituito le immagini dei cartelloni pubblicitari con quelle del paesaggio che si incontra percorrendo la Gene Autry Trail nella Coachella Valley. 
Adesso era contento di aver pagato il suo debito con la giustizia, perché questo l'aveva abituato a considerarsi allo scoperto, sempre e dovunque. [...] Allora l'uomo con un occhio solo [...] le ha detto come tutte le cose appaiano diverse sentendosi allo scoperto quando non c'è più il pensiero di potersi nascondere e così salvare da qualche parte. (Celati 2016: 835)

Si tratta di un altro ribaltamento di prospettive, connesso al nuovo inizio da cui può ripartire l'uomo che ha appena riconquistato la propria libertà e che non ha più voglia di nascondersi dal mondo ma lo vuole affrontare con tutta la sincerità e l'inadeguatezza di «considerarsi allo scoperto, sempre e dovunque». Se si tolgono i paraocchi che limitano lo sguardo, ci si rende conto che tra uomo e paesaggio non c'è separazione ma inevitabile incomprensione, anzi è proprio la visione quotidiana delle cose a renderle estranee e proprio per questo da riscoprire ininterrottamente. Lo ha ben puntualizzato Celati durante la discussione finale al convegno di Salisburgo del 2000, replicando alla relazione di Helmut Meter su «Spazio, sensazione e scrittura in Verso la foce»:

Non c'è un soggetto della visione e poi la cosa vista separata da lui, ma un campo diffuso di cui facciamo parte assieme alla cosa percepita. Se si smette di dare per scontata la separazione tra il soggetto e la cosa percepita, si può pensare il fenomeno del vedere e dell'ascoltare in modo diverso. Cioè in un modo dove il vedere e la cosa vista, il sentire e le cose sentite fanno parte dello stesso fenomeno. (Celati 2002b: 181)

Rifacendosi al concetto di Spinoza per cui l'altro da sé è solo immaginario e alla fenomenologia di Merleau-Ponty, Celati delinea il principio che sta alla base dei suoi racconti di descrizione, ovvero l'immersione percettiva ed emozionale nel paesaggio in una stretta continuità fenomenica che allo stesso tempo destabilizza e acuisce i sensi. Non si tratta né di una romantica proiezione dell'animo del soggetto sul mondo né del suo contrario - come aveva concluso Meter: «lo spazio esterno incide dunque sullo spazio interno e l'osservazione del mondo circostante riporta l'attenzione all'osservatore quale paradigma antropologico» (Meter 2002: 116).

Si comprende, dunque, il motivo per cui nei diari-racconti di Verso la foce la riflessione sul paesaggio padano scaturisca sempre dall'osservazione di una luce inattesa e inclassificabile; come accade in Un paesaggio con centrale nucleare, quando l'osservazione prolungata sulla superficie delle acque del Po di «sparsi raggi di sole [che] portano un brillio che mi dà lievi stralunamenti» lo conduce ad una riflessione sul disorientamento e sull'inconsistenza del cosiddetto «mondo reale»; proiettandolo quasi in un paesaggio lacustre di un tardo Constable, Branch Hill Pond (1828) o di Turner, Landscape with a River (1845):

Deperibilità svelta del cosiddetto «mondo reale», non si distingue bene da un miraggio. Per forza l'intelligenza arriva sempre in ritardo: non lo capisce proprio tutto questo passare e perdersi nell'incerto, la dimenticanza che dovunque ci avvolge e ci porta. (Celati 2016: 1022)

Si giunge così fino alla resa della scrittura nei confronti dello sguardo, incapace di descrivere il mondo, di catturare l'inafferrabilità della visione, come 
invece riesce a fare in qualche modo la pittura; resa che risulta palese nella conclusione del racconto Verso la foce, che dà il titolo alla raccolta:

Pretese delle parole; pretendono di regolare i conti con quello che succede là fuori, di descriverlo e definirlo. Ma là fuori tutto si svolge non in questo o in quel modo, c'entra poco con ciò che dicono le parole. Il fiume qui sfocia in una distesa senza limiti, i colori si mescolano da tutte le parti: come descrivere? [...] Smettiamola: il buco dove tutto scompare è qui dove sono, ingorgato dal sentimento di tutti quelli che se ne sono andati prima di me. Sono qui alle foci del Po e penso a loro. D'un tratto risuonano richiami di gabbiani, uno chiama e altri rispondono. Anche le parole sono richiami, non definiscono niente, chiamano qualcosa perché resti con noi. E quello che possiamo fare è chiamare le cose, invocarle perché vengano a noi con i loro racconti: chiamarle perché non diventino tanto estranee da partire ognuna per conto suo in una diversa direzione del cosmo, lasciandoci qui incapaci di riconoscere una traccia per orientarci. (Celati 2016: 1092-93) ${ }^{6}$

Il paesaggio sfugge alla descrizione, alla pretese della parole di raccontare uno spazio «senza limiti» dai colori indeterminati tra cielo, acqua e terra. Questa immagine può ricordare le marine di Constable, ma sopratutto quelle di Turner nelle quali l'osservatore non sta solo guardando il paesaggio ma fa parte di quel luogo, portandoci in uno spazio della rappresentazione in cui - usando le parole di Celati - «il vedere e la cosa vista, il sentire e le cose sentite fanno parte dello stesso fenomeno». Anche se il luogo osservato è familiare o apparentemente di facile lettura, l'osservatore non assuefatto alla consuetudine della visione riesce a cogliere la miriade di sfumature che lo rendono sempre inatteso e irriducibile ad una descrizione univoca - si veda il Sunset Sky above a Landscape (1820-25) di Turner o l'informale Paesaggio (1964) di Morlotti.

In conclusione, non si può che volgere lo sguardo alla figura del pittore d'insegne Emanuele Menini, che in qualità di grande conoscitore delle Condizioni di luce sulla Via Emilia è alla ricerca della luce perfetta e immobile per fissare il paesaggio padano una volta per tutte. Si tratta di un desiderio delirante che lo conduce alla morte in mezzo alla neve, proprio nel momento in cui ha trovato la luce ideale per dipingere i colori e le ombre, senza la fastidiosa interferenza della «disfazione» luminosa prodotta dai riflessi artificiali e dai gas di scarico che fa vibrare le cose. Secondo l'analisi qui proposta, il pittore Menini può essere considerato non solo un acuto osservatore della realtà che riesce a percepire la luce al di là della coltre di assuefazione (il «guardare categoriale») a cui costringe il regime scopico della desolazione, ma anche uno strenuo difensore del ritorno all'ordine, alla ricerca della luce perfetta attraverso cui rappresentare il paesaggio nella sua essenza e alleviare il dolore per la «disfazione» del mondo. Se Celati può identificarsi con Menini per quanto riguarda il primo aspetto, nell'ottica di un ribaltamento copernicano dello sguardo, di una sorta di «estetica del reincanto» (Rizzante 1993: 33-34), di certo non è interessato a fornire una rappresentazione univoca del paesaggio lì dove non è possibile. Lo scrittore cerca invece di

6 Su questi temi vedi anche le dichiarazioni di Celati contenute ne «La trasparenza delle immagini» (Celati 2010: 145-149). 
osservare lo spazio attorno a sé proprio nella sua indecidibile discrepanza tra la possibilità di catturare frammenti di esperienza estatica e il naufragare nell'irriducibile complessità della percezione immersiva nel paesaggio.

\section{Bibliografia}

Arcangeli, F., 1976, «Romanticismo, neoclassicismo, informale, razionalità», in Id., Dal romanticismo all'informale. Lezioni 1970-71, Bologna, Alfa Edizioni.

Arcangeli, F., 1977, «Lo spazio romantico», in Id., Dal romanticismo all’informale, Torino, Einaudi.

Celati, G., 1986, «Un sistema di racconti sul mondo esterno», Quindi, gennaio, Dal fiume al mare. Esplorazioni lungo la Via Emilia, p. 6-9.

Celati, G., 2002a, «Ultimi contemplatori», IBC, 3, 1997; poi in Sitta (a cura di), 2002, p. 203-207.

Celati, G., 2002b, «Voci sparse. Frammenti di un dibattito», in Kuon (a cura di), 2002, p. 177-202.

Celati, G., 2003, «Collezione di spazi», il verri, 21, p. 57-92.

Celati, G., 2008, «Di cosa si parla quando si parla di paesaggi», Riga, 28, Gianni Celati, sezione extra online.

Celati, G., 2010, «La trasparenza delle immagini», in Jones R., Hinterland. La bassa bolognese: un'identità in trasformazione, Bologna, Artiere, p. 145-149.

Celati, G., 2011, Conversazioni del vento volatore, Macerata, Quodlibet.

Celati, G., 2016, Romanzi, cronache e racconti, a cura di M. Belpoliti e N. Palmieri, Milano, Mondadori.

Clark, K., 1985, Il paesaggio nell'arte, con una nota di A. Emiliani, Milano, Garzanti.

Kuon, P. (a cura di), 2002, Voci delle pianure, Atti del convegno di Salisburgo, 23-25 marzo 2000, Firenze, Cesati.

Iacoli, G., 2002, Atlante delle derive. Geografie da un'Emilia postmoderna: Gianni Celati e Pier Vittorio Tondelli, Reggio Emilia, Diabasis.

Meter, H., 2002, «Spazio, sensazione e scrittura in «Verso la foce» di Gianni Celati», in Kuon (a cura di), 2002, p. 105-116.

Raimondi, E., 2002, "Sentire un luogo», in Sitta (a cura di), 2002, p. 13-16.

Rizzante, M., 1993, Il geografo e il viaggiatore: variazioni su I. Calvino e G. Celati, Fossombrone, Metauro.

Sitta, C. A. (a cura di), 2002, L'orizzonte di bruma. Luoghi del Novecento poetico in Emilia: Modena, Reggio Emilia, Parma, Piacenza, Atti del Convegno, premessa di E. Raimondi, Modena, Edizioni del laboratorio.

Spadoni, C. (a cura di), 2006, Turner Monet Pollock: dal romanticismo all'informale. Omaggio a Francesco Arcangeli, catalogo della mostra tenutasi al Museo d'arte della città di Ravenna, 19 marzo-23 luglio 2006, Milano, Electa.

Weelen, G., 1984, Turner. Le forme della luce, Milano, Jaca Book. 